\title{
Utilização de um software para organização e integração de tarefas
}

DOI: 10.37702/2175-957X.COBENGE.2021.3459

Victor da Silva Magalhães - victor.magalhaes@engenharia.ufjf.br Universidade Federal de Juiz de Fora

Rua orestes homem da costa 189

28470-000 - Santo Antônio de Pádua - RJ

Angelina Kneip Figueiredo - kneip.figueiredo@engenharia.ufjf.br Universidade Federal de Juiz de Fora

Melo Viana 191

36600-000 - Bicas - MG

Arthur Fernandes Rezende - arthur.rezende@engenharia.ufjf.br

Universidade Federal de Juiz de Fora

Rua Tiradentes 672

36015-360 - Juiz de Fora - MG

Lorrayne Nogueira Pereira Duarte - lorrayne.duarte@engenharia.ufjf.br Universidade Federal de Juiz de Fora

Rua Adalgisa Gonçalves Soares 210

36037-390 - Juiz de Fora - MG

Lavínia Naval Silva - lavinia.silva@engenharia.ufjf.br

Universidade Federal de Juiz de Fora

Rua Coronel João Rodrigues Vicente 756

36170-000 - Piraúba - MG

Roberta Cavalcanti Pereira Nunes - rcpnunes@gmail.com

Universidade Federal de Juiz de Fora

Rua Oscavo Gonzaga Prata 372

36033-220 - Juiz de Fora - MG

Resumo: O Scrum é uma metodologia ágil para gestão e planejamento que auxilia na execução de projetos. Com o intuito de auxiliar a implementação dele dentro do Grupo de Educação Tutorial de Engenharia de Produção (GET-Produção) da 
Universidade Federal de Juiz de Fora (UFJF), bem como tornar a gestão das atividades mais visual, o segmento utiliza um software de organização e o adapta para o ambiente em que está inserido. O presente trabalho tem o intuito de detalhar a utilização desse no grupo, assim como discutir a eficiência dele juntamente com uma gestão ágil. Para a coleta de dados, que incluiu membros ativos e egressos do GET-Produção, foi utilizado um formulário online. Por fim, o trabalho pretende sugerir a implementação de tais ferramentas a outros Programas de Educação Tutorial do Brasil.

Palavras-chave: Gestão, Scrum, Trello 


\section{Utilização de um software para organização e integração de tarefas}

\section{INTRODUÇÃO}

Segundo Soares (2004), as metodologias ágeis tem como característica serem adaptativas ao invés de preditivas, possibilitando que se adaptem a novos fatores decorrentes do desenvolvimento do projeto, ao invés de analisar previamente tudo o que pode acontecer no decorrer do desenvolvimento. Porém, de acordo com Cohn (2011), a transição para os métodos ágeis é mais difícil do que as empresas esperam e as mudanças exigidas para a obtenção de todas recompensas que ser ágil pode trazer são difíceis de alcançar.

A exemplo de empresas como Google, Amazon e pequenas startups (SUTHERLAND, 2014), as atividades realizadas pelo GET-Produção estão pautadas na utilização da metodologia Scrum que, segundo Satpathy (2016), está entre as mais populares das chamadas metodologias ágeis. A metodologia foi criada para auxiliar no desenvolvimento de softwares. Porém, percebeu-se, com o passar do tempo, que ela poderia ser utilizada em qualquer empresa que deseje implementar processos que utilizem gerenciamento de projetos (SILVA, 2010).

Para Bissi (2007), o Scrum é uma metodologia ágil e flexível, que objetiva definir um processo de desenvolvimento iterativo e incremental podendo ser aplicado a qualquer produto ou no gerenciamento de qualquer atividade complexa, além de poder ser utilizado em projetos de grande ou pequeno porte. Visto isso, o GET-Produção utiliza o Scrum para o gerenciamento de projetos em conjunto com a ferramenta Trello. De acordo com Mello (2018), a ferramenta apresenta interface simples, intuitiva e pode ser utilizada por uma ou mais pessoas simultaneamente como uma equipe, com disponibilidade em todos dispositivos com acesso à internet e possuindo inclusive uma versão gratuita, além disso, pode ser personalizada para organizar quaisquer atividades a serem efetuadas.

O GET-Produção utiliza o software Trello para exibir as tarefas que necessitam ser feitas para todo o grupo, bem como marcar reuniões e adicionar pautas para discussão. A interface do aplicativo auxilia a manter a gestão à vista, e facilita o lembrete de datas de entrega, além de possibilitar a escolha dos membros responsáveis por cada atividade. Como uma forma de deixar os projetos mais visíveis dentro do GET-Produção, o grupo optou por utilizar o Scrum, também dentro do Trello. Assim, a manutenção do fluxo de atividades ficou mais fácil de ser feita, pois os membros conseguem visualizá-lo a qualquer momento, bem como atualizá-lo.

Sendo assim, o presente artigo tem como objetivo avaliar a maneira como o Scrum, utilizado com auxílio do software Trello, insere-se como peça fundamental no gerenciamento das atividades do GET-Produção.

\section{METODOLOGIA}

A partir do destaque do objetivo deste artigo, que busca entender como a gestão ágil, em união ao software Trello é capaz de ser eficaz para o gerenciamento de projetos e atividades do GET-Produção UFJF, entende-se que o tipo de pesquisa, quanto aos procedimentos adotados, configura o presente trabalho como um levantamento, que, segundo GIL (2017, p.37), caracteriza-se "[...] pela interrogação direta das pessoas cujo comportamento se deseja conhecer". Com isso, através de uma coleta e análise de dados entre os integrantes do Grupo, pode-se obter conclusões a respeito do problema tratado. 
Dessa forma, o grupo responsável por esse levantamento realizou um questionário online através da ferramenta Formulários Google, a fim de garantir maior praticidade no envio e coleta das respostas, cujas notas foram realizadas numa escala de 01 a 10 . Assim sendo, este levantamento contou com a participação de 21 membros do GET-Produção, dentre eles 8 ativos e 13 egressos.

Dentre as questões abordadas na pesquisa, as de maior destaque que serão aprofundadas e discutidas mais adiante são: a eficiência do aplicativo online para gestão dos projetos internos do grupo; sua influência na gestão horizontal; sua aplicabilidade em outros Grupos e Programas de Educação Tutorial; e a facilidade de se implementar a metodologia ágil para gestão de projetos.

\section{RESULTADOS E DISCUSSÃO}

Através dos resultados da pesquisa, a qual teve 21 respostas, consegue-se perceber a importância da ferramenta Trello e da metodologia Scrum para a equipe de projeto. Com esses dois mecanismos é possível perceber que o gerenciamento do projeto se torna mais eficiente, pois há uma melhor definição e gestão das ações, por exemplo, garantindo uma otimização e uma melhor administração do projeto e também da organização.

Os gráficos das figuras 1 e 2 apresentam uma avaliação dos membros do grupo sobre o quanto o Trello e o Scrum, respectivamente, os auxiliaram na realização dos projetos do GET. Pode-se observar, em ambos os gráficos, que notas de 8 a 10 são maioria. O gráfico sobre a eficácia do trello apresenta $90,47 \%$ das notas acima de 9 e o que avalia o Scrum apresenta $90,48 \%$ das notas acima de 8.

Figura 1: Auxílio do Trello em projetos.

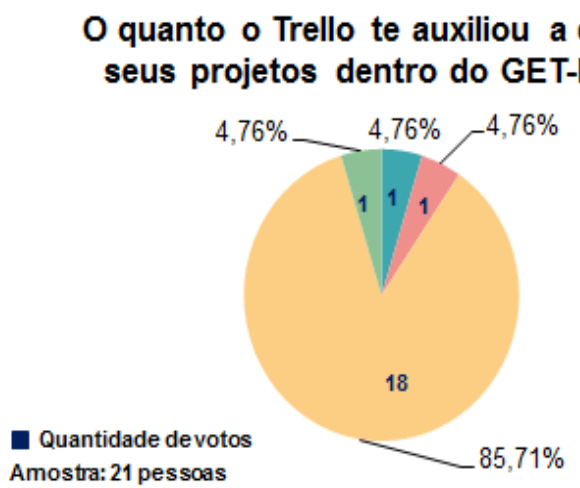

Fonte: Os autores (2021).
Figura 2: Auxílio do Scrum em projetos.

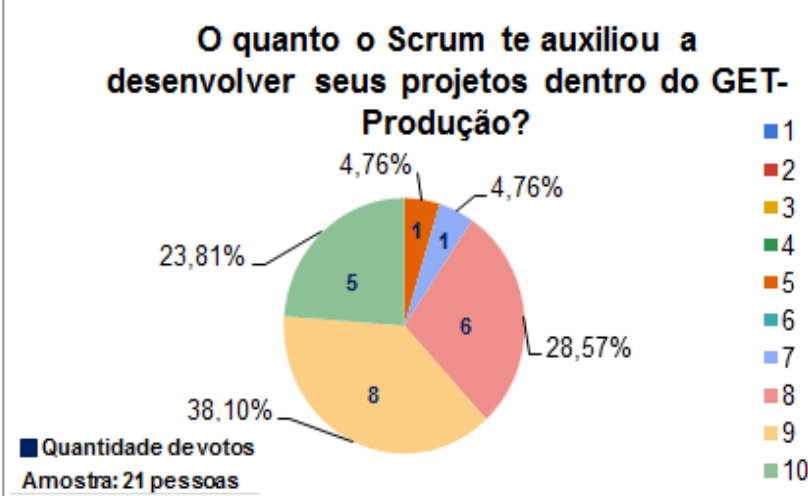

Fonte: Os autores (2021).

Os PETs/GETs prezam sempre pela horizontalidade, indispensável para o Programa. Visando analisar se o aplicativo Trello auxilia o grupo a ir de encontro a essa diretriz, a partir do gráfico da figura 3 pode-se concluir que o Trello pode ser uma ferramenta muito eficaz, com $85,72 \%$ das notas acima de 9 . Isso se dá pois o aplicativo permite total acesso a quaisquer informações por todos os membros, bem como alterações em tempo real. 


\section{COBENCE de Educação em Engenharia 2021 da ABENGE \\ 28 a 30 de SETEMBRO \\ Evento Online \\ "Formaçāo em Engenharia: \\ Tecnologia, Inovação e Sustentabilidade"}

Figura 3: Auxílio do Trello para horizontalidade.

O quanto a interface da ferramenta Trello auxilia em uma gestão horizontal, dentro do GET-Produção?

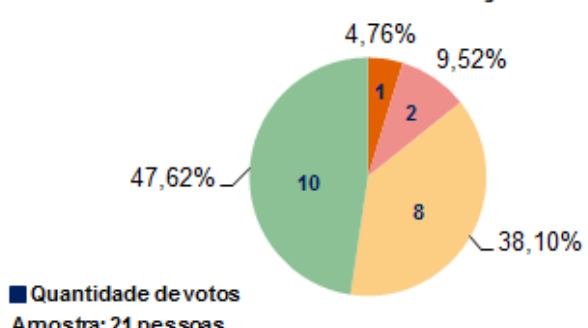

Fonte: Os autores (2021).
Figura 4: Facilidade de unir Scrum e Trello.

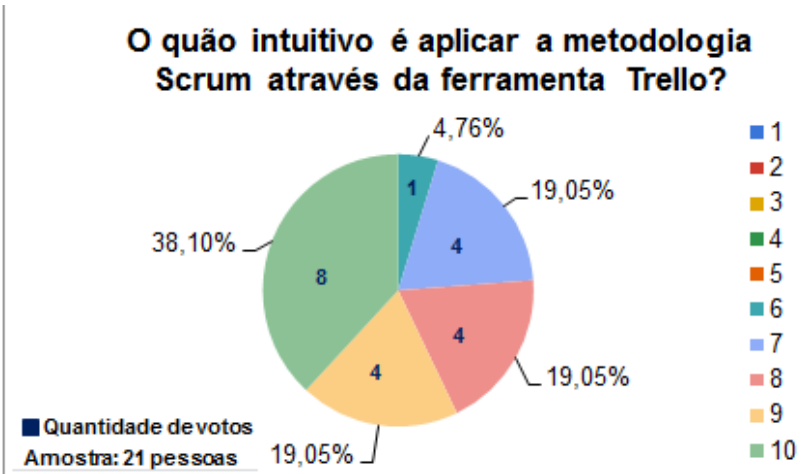

Fonte: Os autores (2021).

Com o objetivo de demonstrar a eficácia de uma gestão ágil junto ao Trello, apresenta-se o gráfico da figura 4, o qual analisa se através do Trello é possível aplicar a metodologia Scrum com maior facilidade, uma vez que, neste software, é possível organizar algumas etapas do Scrum, como dividir processos em microprocessos, garantindo a execução de Backlogs do Produto, que segundo Ken Schwaber (2009, p.5) "é uma lista priorizada de tudo que pode ser necessário no produto" onde considera-se o "produto" sendo o projeto. A ferramenta ainda permite adicionar os responsáveis por essas tarefas, bem como a data de entrega. Do gráfico 4 , observa-se que $76,2 \%$ das notas foram acima de 8, o que comprova a eficácia da junção das ferramentas no grupo.

Figura 5: Alcance dos resultados com o Trello.

O qual importante a ferramenta Trello foi, e ainda é, para o GET-Produção alcançar seus resultados?

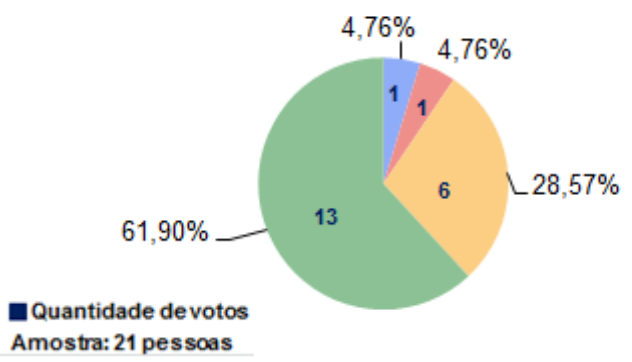

Fonte: Os autores (2021).

$$
\begin{aligned}
& \square 1 \\
& \square 2 \\
& \square 3 \\
& \square 4 \\
& \square 5 \\
& =6 \\
& =7 \\
& =8 \\
& =9 \\
& =10
\end{aligned}
$$

Figura 6: Aplicabilidade do Trello.

Para você, a ferramenta Trello poderia ser aplicada, com sucesso, em qualquer grupo PET/GET do Brasil (fora da Engenharia de Produção)?

Quantidade devotos Amostra: 21 pessoas

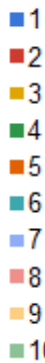

Fonte: Os autores (2021). 
Através dos gráficos das figuras 1 a 6, percebe-se que, de fato, o Trello é uma ferramenta que auxilia de forma prática ao GET-Produção a alcançar seus resultados. O gráfico da figura 5, cujo objetivo é analisar a importância do Trello para o alcance dos resultados dos projetos do grupo, obteve 95,24\% das notas acima de 8 .

$\mathrm{Na}$ figura 6, traz-se o gráfico com a opinião da amostra sobre a aplicação da ferramenta em outros PET's do Brasil, no qual 95,23\% das notas são acima de 8 também e, então, segundo a pesquisa, fica evidente que a aplicação da metodologia ágil através do Trello, neste caso, poderia se tornar efetiva em outros grupos em outros segmentos educacionais do Brasil.

Levando em consideração que a duração média de membros no GET-Produção é de 1 ano, percebe-se, ainda, que as ferramentas conseguem driblar a rotatividade dos grupos e auxiliar na gestão de conhecimento, uma vez que consegue-se analisar períodos de tempos anteriores, funções estabelecidas, backlogs e prazos, para que os projetos continuem em andamento durante e após as trocas de membros.

\section{CONSIDERAÇÕES FINAIS}

Com os resultados do presente trabalho, percebe-se que a metodologia Scrum aliada ao software Trello é avaliada pelos participantes do GET-Produção UFJF como um fator imprescindível no gerenciamento dos projetos e outras atividades realizadas pelo grupo, pois contribui para a construção de uma gestão horizontal e auxilia na gestão do conhecimento.

No modelo de gestão horizontal não há níveis hierárquicos e as decisões são tomadas em conjunto com os colaboradores, e para isso é necessário que haja transparência sobre as ações de cada um. Para alcançar tal transparência e contribuir para a gestão, o Grupo utiliza o aplicativo Trello. Como visto nos resultados da pesquisa, a maior parte dos colaboradores acredita que o aplicativo auxilia a gestão horizontal, pois nele é possível o acesso de todas as informações por todos os membros.

Essas informações são armazenadas para análises e consultas posteriores, o que é vantajoso para combater e minimizar a perda de conhecimento, que é ocasionada pela rotatividade dos integrantes. Dessa forma, manter os dados dos ciclos anteriores e o conhecimento adquirido através deles auxilia a compor a gestão do conhecimento do Grupo, uma vez que as boas práticas, criadas e/ou aprimoradas, pelos colaboradores daquele período perdurem na organização e possam ser ensinadas aos novos membros.

Outro benefício advindo da boa gestão do conhecimento feita no software Trello embasada na metodologia ágil é a rápida adaptação dos novos participantes à rotina de atividades do GET-Produção. Por meio das colunas A fazer/Fazendo/Feito é possível visualizar rapidamente quais tarefas devem ser feitas durante a semana, o prazo para realizá-las e os responsáveis por cumpri-las. Tais colunas são originadas do método Scrum e a pesquisa nos permite constatar que a maioria dos membros e egressos considera que essa metodologia os ajudou a desenvolver projetos.

Desse modo, com base nos resultados da pesquisa e nos benefícios adquiridos com a utilização do Scrum em conjunto com o Trello, pode-se constatar que tais ferramentas agregam eficiência no gerenciamento de quaisquer projetos, podendo, portanto, ser aplicadas em PET's de todo o Brasil. Ademais, o trabalho corrobora com as práticas de ensino superior ao apresentar ferramentas que promovam o aprendizado de forma ativa.

Em trabalhos futuros, o Grupo pretende assessorar os demais segmentos educacionais da Faculdade de Engenharia da UFJF na implementação de boas práticas 
de gestão, como exemplo as supracitadas neste artigo, empregadas pelo GET-Produção de forma a comprovar os resultados expostos.

\section{Agradecimentos}

Os autores responsáveis por este artigo reconhecem todo incentivo, suporte e colaboração do GET-Produção, ao gerar um ambiente propício para a elevação tanto pessoal quanto profissional de seus membros. Vale salientar também o agradecimento à Universidade Federal de Juiz de Fora, por proporcionar essa oportunidade aos autores.

\section{REFERÊNCIAS}

BISSI, Wilson. Scrum - Metodologia de Desenvolvimento Ágil, Campo Mourão, 2007. GIL, A. C. Como elaborar projetos de pesquisa. 6.ed. São Paulo: Atlas, 2017.

COHN, Mike. Desenvolvimento de Software com Scrum. 1 ed, Porto Alegre: Bookman, 2011.

MELLO, Anna Carolina; SOUZA, Luiz Henrique Gomes de. Solução Simplificada para o Monitoramento e Controle de Projetos Utilizando a Ferramenta Trello. Boletim do Gerenciamento, [S.I.], v. 2, n. 2, 2018.

SATPATHY, Tridibesh. Um Guia Para o Conhecimento em Scrum. 1. ed. VMEdu: [s. n.], 2016.

SILVA, M. A. C.; Roriz Filho, H.; Silva, H. F. N. Análise do BA durante o Processo Scrum. XVII Simpósio de Engenharia de Produção. Bauru, 2010. Anais. Disponível em: https://simpep.feb.unesp.br/anais_simpep.php?e=5. Acesso em: 15 abr. 2021.

SOARES, M. S.; Metodologias Ágeis Extreme Programming e Scrum para o Desenvolvimento de Software. Revista Eletrônica de Sistemas de Informação - RESI. v. 3, n.1, p. 1-2, 2004. Online. Disponível em: http://periodicosibepes.org.br/index.php/reinfo/article/view/146/38. Acesso em: 15 abr. 2021.

SUTHERLAND, Jeff. SCRUM: A arte de fazer o dobro do trabalho em metade do tempo. 1. ed. rev. [S. I.]: LeYa, 2014.

\section{USE OF AN SOFTWARE FOR ORGANIZATION AND INTEGRATION OF TASKS}

Abstract: The Scrum is an agile methodology for administration and planning that helps in the projectes's execution. With the intention of helping the his implementation of it inside the Tutorial Education Group in Product Engineering (GET-Produção) from University Federal de Juiz de Fora, as well as make the administration more visual, the segment uses a organization software and adapt it for the environment where it is inserted. The present work has the intention of detailing the use of it in the group, as well as discussing 
the efficiency of it along with a agil administration. For the data collect, which included active and egressed members from GET-Produção, an online form was used. Lastly, the work pretends to suggest the implementation of these tools to others Tutorial Educations Programs from Brazil.

Keywords: Administration. Scrum. Trello. 